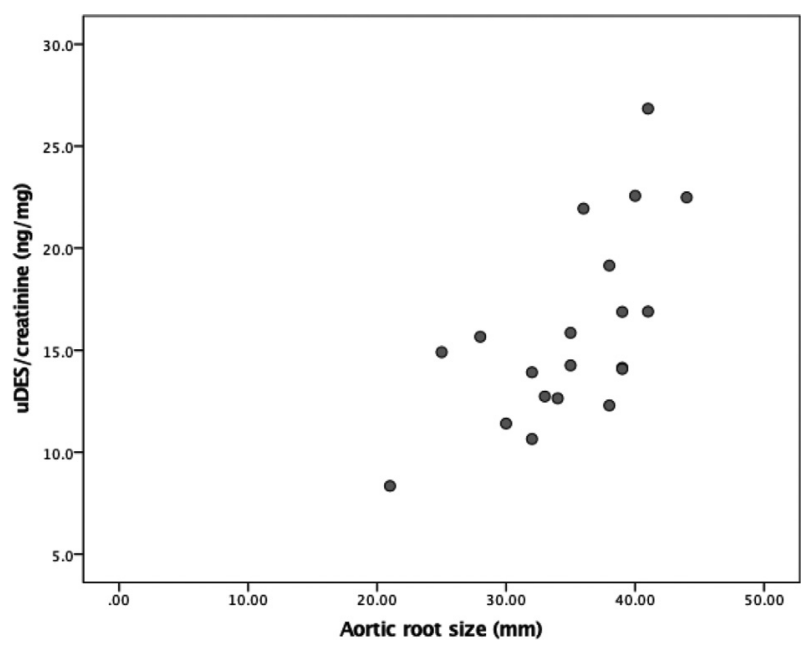

Abstract 126 Figure 1 Correlation between uDES (ng/mg creatinine) and aortic root size $(\mathrm{mm})$

patients with larger aortic roots. This suggests a potential utility of desmosine as a biomarker in patients with BAV. Larger studies are needed to test this hypothesis.

Conflict of Interest None

\section{DEVELOPMENTAL ROCK DOWNREGULATION DISRUPTS SARCOMERIC STRUCTURE RESULTING IN THE DEVELOPMENT OF HYPERTROPHIC CARDIOMYOPATHY}

${ }^{1}$ Kate Bailey*, ${ }^{2}$ Guy MacGowan, ${ }^{1}$ Simon Tual-Chalot, 'Lauren Phillips, ${ }^{3}$ Timothy J Mohun, ${ }^{1}$ Deborah J Henderson, ${ }^{1}$ Helen Arthur, ${ }^{1}$ Simon Bamforth, ${ }^{1}$ Helen M Phillips. ${ }^{1}$ Newcastle University; ${ }^{2}$ Freeman Hospital; ${ }^{3}$ Francis Crick Institute

\subsection{6/heartjnl-2019-BCS.124}

Introduction Congenital heart defects are common, affecting $\sim 1 \%$ of live births, while adult heart disease is the main cause of death in the UK. Defects acquired during foetal development can have a lasting detrimental effect on adult heart function. Therefore, understanding the underlying mechanisms involved in cardiac development and disease progression are of particular importance.

Cardiomyopathy is a disease of the heart muscle. Hypertrophic cardiomyopathy (HCM) is one of the most common forms of cardiomyopathy; mutations have been identified in the troponin complex, a key component of the sarcomere, signifying that disruption to sarcomeric integrity plays an important role in disease progression.

Rho Kinase (ROCK) is expressed in the heart during development and is known to be a regulator of actin-myosin contraction through the phosphorylation of the troponin complex. Our aim was to determine the effect of developmental ROCK downregulation on sarcomeric integrity and impact this had on the function of the adult heart.

Methods Cre-LoxP technology was utilised to create a conditional mouse model in which ROCK was specifically downregulated in the cardiomyocytes from E9.25, during cardiac development. Histological techniques were used to assess the embryonic and adult heart phenotype. Analysis of the cytoarchitecture was performed by TEM while cine cardiac MRI was used to assess overall adult heart function.

Results and Conclusions: Developmental downregulation of ROCK in the cardiomyocytes resulted in loss of sarcomere integrity at E10.5, associated with a reduction in the levels of phosphorylation of cardiac Troponin I and $\mathrm{T}$, and reduced cardiomyocyte proliferation at E11.5. This caused abnormal myocardial wall development, where the compact myocardium failed to thicken. The impact of these embryonic abnormalities, triggered compensatory foetal cardiomyocyte hypertrophy, which persisted throughout postnatal development and into adult life. Over time this continued hypertrophy became detrimental, triggering cardiac remodelling. Mutants exhibit key features of HCM including concentric hypertrophy with systolic dysfunction, fibrosis and re-expression of foetal genes. This data suggests a novel developmental origin of the sarcomeric phenotype of HCM and indicates disruption in ROCK signalling may contribute to the pathogenesis of HCM.

(Bailey et al., Disruption of embryonic ROCK signalling reproduces the sarcomeric phenotype of Hypertrophic Cardiomyopathy. JCI Insight, in press)

Conflict of Interest None

\section{THE IMPACT OF AORTIC VALVE REPLACEMENT ON SURVIVAL IN PATIENTS WITH NORMAL FLOW LOW GRADIENT SEVERE AORTIC STENOSIS: A PROPENSITY- MATCHED COMPARISON}

${ }^{1}$ Roxy Senior*, ${ }^{2}$ Sahrai Saeed, ${ }^{3}$ Anastasia Vamvakidou, ${ }^{4}$ Rajdeep Khattar, ${ }^{4}$ Wei Li. ${ }^{1}$ Royal Brompton Hospital and National Heart and Lung Institute, Imperial College, London; ${ }^{2}$ Haukeland University Hospital, Bergen, NO; ${ }^{3}$ Northwick Park Hospital; ${ }^{4}$ Royal Brompton Hospital

\subsection{6/heartjnl-2019-BCS.125}

Introduction To assess the survival benefit of aortic valve replacement (AVR) in patients with normal flow low gradient severe aortic stenosis (AS).

Methods A retrospective study of prospectively collected data of 276 patients (mean age $75 \pm 15$ years, 51\% male) with normal transaortic flow (flow rate [FR] $200 \mathrm{ml} / \mathrm{s}$ or stroke volume index [SVi] $35 \mathrm{ml} / \mathrm{m}^{2}$ ) and severe AS (aortic valve area [AVA] $<1.0 \mathrm{~cm}^{2}$. The outcome measure was all-cause mortality.

Results Of the 276 patients, 55\% $(\mathrm{n}=151)$ were medically treated while $45 \%(n=125)$ underwent an AVR. Over a mean follow-up of $3.2 \pm 1.8$ years (range 0-6.9 years) a total of $96(34.8 \%)$ deaths occurred: $17(13.6 \%)$ in AVR group versus $79(52.3 \%)$ in those medically treated, when transaortic flow was defined by FR $(\mathrm{p}<0.001)$ (figure 1$)$. When transaortic flow was defined by SVi, a total of $79(31.3 \%)$ deaths occurred: $18(15.1 \%)$ in AVR group versus 61 $(45.9 \%)$ in medically treated $(\mathrm{p}<0.001)$. In a propensitymatched multivariable Cox regression analysis adjusting for age, gender, body surface area, smoking, hypertension, diabetes mellitus, atrial fibrillation, peripheral vascular disease, chronic kidney disease, left ventricular ejection fraction, left ventricular mass and mean aortic gradient, not having AVR was associated with a 6.3 fold higher HR of all-cause mortality (HR 6.28; 95\% CI 3.34-13.16, p<0.001) when flow was defined by FR. In the SVi-guided model it was 3.83 fold (HR 3.83; 95\% CI 2.30-6.37, p<0.001).

Conclusion In patients with normal flow low gradient severe AS, AVR was associated with a significantly improved survival compared to those who received standard medical treatment. Conflict of Interest No 